\title{
Cardiovascular $\beta$-adrenoceptor sensitivity of undernourished subjects
}

\author{
BY M. PREM JAYARAJAN AND P.S. SHETTY* \\ Department of Physiology, Nutrition Research Centre (ICMR), St John's Medical \\ College, Bangalore 560 034, India
}

(Received 3 September 1986 - Accepted 27 January 1987)

1. Eleven normal-weight male subjects (weight/height ${ }^{2}\left(\mathrm{~W} / \mathbf{H}^{2}\right)$ 19.0-22.5) from a good socio-economic background and on ad lib. food intake, and eight undernourished male labourers $\left(\mathrm{W} / \mathrm{H}^{2} 16 \cdot 4-18.6\right)$ on low energy intakes, were studied.

2. Comparison of cardiovascular responses to increasing single doses of the $\beta$-agonist, isoproterenol, showed a significantly greater positive chronotropic dose response in the undernourished subjects.

3. Cardiovascular responses to head-up tilt were similar in both the normal-weight and undernourished subjects.

4. Undernourished individuals may show an increase in $\beta$-adrenoceptor sensitivity which may be akin to a denervation type of supersensitivity as a result of a nutrition-related reduction in sympathetic activity.

Catecholamine sensitivity may reflect the nutritional status of an individual, since the activity of the sympathoadrenal system is enhanced on increasing energy intake (Young \& Landsberg, 1977a) and declines following energy restriction in animals (Young \& Landsberg, 1977b) and man (Shetty et al. 1979). Adrenergic receptor numbers and their sensitivity have been shown to undergo modulation in response to changes in circulating catecholamine levels (Motulsky \& Insel, 1982). Supersensitivity or up-regulation as well as down-regulation of $\alpha$ - and $\beta$-receptors has been known to occur in response to altered agonist levels (Colucci et al. 1981; Bristow et al. 1982; Davies et al. 1982; Lefkowitz et al. 1984).

Few studies, however, have addressed the problem of whether adrenergic receptor responsiveness is actually altered in undernutrition (Crandall et al. 1983) especially in man, and to what extent this would modify physiological functioning. The present study was undertaken to examine the responsiveness of myocardial and vascular $\beta$-adrenoceptors in undernourished human subjects on chronically low energy intakes, when tested using the isoproterenol sensitivity test (Cleaveland et al. 1972).

\section{MATERIALS AND METHODS}

Subjects

Nineteen healthy young male volunteers were studied. They were classified on the basis of their body mass index (BMI, weight/height ${ }^{2}$ ) into a normal weight group and an undernourished group. Eleven normal-weight controls (BMI between 19 and 22.5) of mean age 21.3 (SE 0.4) years, from a good socio-economic background, which permitted a normal ad lib. food intake, were compared with eight undernourished labourers (BMI between 16.4 and 18.6) of mean age 26.4 (SE 1.8) years. All eight labourers lead a physically active life while only seven of the eleven normal-weight subjects had daily activity patterns suggestive of moderate activity, while the rest were sedentary individuals.

Each subject gave fully informed and written consent and the study received approval from the Institution's Ethical Approval Committee. 


\section{Methods}

Nutritional status was assessed in all subjects, based on anthropometry: body-weight, height, BMI, sum of four skinfolds (triceps, biceps, subscapular and suprailiac) and the mid-upper-arm circumference. The customary energy intake of each subject was also determined by the $24 \mathrm{~h}$ recall method on two separate occasions. Each subject underwent a complete clinical examination including a twelve-lead electrocardiogram (ECG). Fasting blood samples were obtained for the estimation of haemoglobin by Sahli's method and measurement of total serum thyroxine $\left(\mathrm{T}_{4}\right), 3,5,3^{\prime}$-triiodothyronine $\left(\mathrm{T}_{3}\right)$ (by radioimmunoassay kits from Bhabha Atomic Research Centre, Bombay) and free $T_{3}$ levels (radioimmunoassay kit from Diagnostic Products Corp., Los Angeles, USA).

Cardiovascular $\beta$-adrenoceptor responsiveness was evaluated by the standardized isoproterenol sensitivity test (Cleaveland et al. 1972). The test was performed on each subject after an overnight $12 \mathrm{~h}$ fast, in the same room by the same personnel at room temperatures between 26 and $29^{\circ}$. The subject rested supine while a $21 \mathrm{G}$ butterfly cannula was introduced into a brachial vein and kept patent with a sterile $38 \mathrm{~g}$ citrate/1 lock. After $30 \mathrm{~min}$ four single doses $(0.25,0.5,1.0$ and $2.0 \mu \mathrm{g})$ of isoproterenol hydrochloride (Unichem, Chessington, Surrey) were rapidly injected, with $10 \mathrm{~min}$ intervals between doses. Doses of the $\beta$ agonist were made up to a volume of $1 \mathrm{ml}$ in saline $(9 \mathrm{~g}$ sodium chloride/l) and injected rapidly, followed by $2 \mathrm{ml}$ saline (within $10 \mathrm{~s}$ ). An ECG recording (at $25 \mathrm{~mm} / \mathrm{s}$ ) was made using a Beckman RS Dynograph, before and for 2 min after each dose of isoproterenol. Continuous ECG monitoring on an oscilloscope was carried out to detect any premature ventricular contractions. Blood pressure was also recorded at 0.5 -min intervals (by sphygmomanometer) before and for 2 min following each dose. Cardiovascular responses appeared within $15 \mathrm{~s}$ of each injection and reached a peak by $30-45 \mathrm{~s}$, hence a $2 \mathrm{~min}$ recording was adequate. Subjects received a full explanation of the procedure and were cautioned against breathing deeply after each dose, to avoid the effects of hyperventilation on the heart rate. Dose-response curves for the increase in heart rate and fall in diastolic blood pressure, with increasing doses of isoproterenol were plotted on semilog paper. The chronotropic dose ${ }_{25}\left(\mathrm{CD}_{25}\right)$ producing an absolute increase of 25 beats/min (bpm) above the mean resting heart rate, was also calculated from the dose-response curves.

Cardiovascular responses to $70^{\circ}$ head-up tilt (HUT) was also determined separately in all subjects of both groups. Following a $30 \mathrm{~min}$ rest period in the supine position, each subject was tilted to $70^{\circ}$ over $30 \mathrm{~s}$, using a Tilt table which supported the perineal region and left the feet unsupported. The subject remained tilted for $10 \mathrm{~min}$ and was then returned to the horizontal position. Heart rate and blood pressure were recorded at 2-min intervals both during and after tilting.

\section{Statistical analysis}

The results are expressed as means with their standard errors. Statistical analysis of results was done using the Student's $t$ test for unpaired variables and a two-way analysis of variance (ANOVA). The possibility of occurrence by chance of $P<0.05$ was accepted as being statistically significant.

\section{RESULTS}

The energy intakes and anthropometric values for the normal-weight and undernourished subjects are summarized in Table 1 . The undernourished subjects had significantly lower body-weights, BMI, percentage body fat, mid-upper-arm circumference and triceps skinfolds compared with the controls. Their total daily energy intake was also significantly lower, but the mean energy intake per kg fat-free mass (FFM; calculated using equations 
Table 1. Energy intake and anthropometry of normal-weight and undernourished Indian adult male subjects

(Mean values with their standard errors)

\begin{tabular}{|c|c|c|c|c|}
\hline & \multicolumn{2}{|c|}{ Normal-weight controls $(n 11)$} & \multicolumn{2}{|c|}{ Undernourished subjects $(n$ 8) } \\
\hline & Mean & SE & Mean & $\mathrm{SE}$ \\
\hline Energy intake $(\mathrm{MJ} / \mathrm{d})$ & 9.78 & 0.46 & $6.56^{* * *}$ & 0.40 \\
\hline Energy intake $(\mathrm{kJ} / \mathrm{kg}$ FFM per d) & $191 \cdot 3$ & $9 \cdot 3$ & $169 \cdot 5$ & $8 \cdot 7$ \\
\hline Height (m) & 1.73 & 0.02 & $1.62^{* *}$ & 0.02 \\
\hline Wt $(\mathrm{kg})$ & $61 \cdot 4$ & 1.8 & $44 \cdot 8^{* * * *}$ & 1.8 \\
\hline BMI & 20.5 & 0.3 & $17.0^{* * *}$ & 0.2 \\
\hline Percentage body fat & 16.1 & $0 \cdot 4$ & $13 \cdot 4^{* *}$ & 0.6 \\
\hline MAC (mm) & $267 \cdot 0$ & $5 \cdot 0$ & $232 \cdot 0^{* * *}$ & $4 \cdot 0$ \\
\hline \multicolumn{5}{|l|}{ Skinfold thickness (mm): } \\
\hline Triceps & $8 \cdot 3$ & 0.9 & $5 \cdot 4^{*}$ & 0.6 \\
\hline$\sum 4$ & 37.8 & $4 \cdot 1$ & $26 \cdot 4$ & $2 \cdot 6$ \\
\hline
\end{tabular}

FFM, fat-free mass; BMI, body mass index (weight/height ${ }^{2}$ ); MAC, mid-upper-arm circumference; $\sum 4$, sum of four skinfolds: triceps, biceps, subscapular, suprailiac.

Mean values were significantly different from control values: $* P<0.05, * * P<0.01,{ }^{* * *} P<0.001$.

Table 2. Cardiovascular and biochemical indices for normal-weight and undernourished Indian adult male subjects

(Mean values with their standard errors)

\begin{tabular}{|c|c|c|c|c|}
\hline & \multicolumn{2}{|c|}{ Normal-weight controls ( $n$ 11) } & \multicolumn{2}{|c|}{ Undernourished subjects $(n 8)$} \\
\hline & Mean & SE & Mean & SE \\
\hline Resting heart rate (beats/min) & $60 \cdot 7$ & $2 \cdot 2$ & $58 \cdot 1$ & $2 \cdot 5$ \\
\hline \multicolumn{5}{|l|}{ Supine blood pressure $(\mathrm{mm} \mathrm{Hg})$ : } \\
\hline Systolic & $113 \cdot 0$ & $2 \cdot 4$ & $100 \cdot 6^{* *}$ & $1 \cdot 7$ \\
\hline Diastolic & $75 \cdot 3$ & $2 \cdot 4$ & $71 \cdot 1$ & 1.9 \\
\hline Pulse pressure & 37.6 & $2 \cdot 2$ & $29 \cdot 4^{*}$ & 1.9 \\
\hline Haemoglobin $(\mathrm{g} / 1)$ & $145 \cdot 0$ & 3-0 & $148 \cdot 0$ & $4 \cdot 0$ \\
\hline Serum total $\mathrm{T}_{4}(\mathrm{nmol} / 1)$ & $84 \cdot 7$ & $9 \cdot 5$ & $72 \cdot 2$ & $5 \cdot 3$ \\
\hline Serum total $\mathrm{T}_{3}(\mathrm{nmol} / 1)$ & $2 \cdot 0$ & $0 \cdot 3$ & 1.5 & $0 \cdot 1$ \\
\hline Serum free $T_{3}(\mathrm{pmol} / 1)$ & 4.9 & 0.4 & 4.9 & $0 \cdot 3$ \\
\hline
\end{tabular}

$T_{4}$, thyroxine; $T_{3}, 3,5,3^{\prime}$-triiodothyronine

Mean values were significantly different from control values: ${ }^{*} P<0.05,{ }^{* *} P<0.01$

of Durnin \& Womersley (1974)), although $11.4 \%$ lower than that of the control group, was not statistically significant. All subjects were euthyroid, serum $T_{4}$ and free $T_{3}$ levels being similar in the two groups (Table 2). The total serum $\mathrm{T}_{3}$ was lower in the undernourished subjects, but this was not statistically significant. The mean percentage ratio, serum total $\mathrm{T}_{3}(\mu \mathrm{g} / \mathrm{l})$ : total $\mathrm{T}_{4}(\mu \mathrm{g} / \mathrm{l})$ was lower $(1.75$ (SE 0.09)) in the undernourished subjects compared with the normal-weight controls $(2 \cdot 10$ (SE 0.40$)$ ) but this difference was not statistically significant. Resting heart rates were similar in the normal and undernourished groups. The undernourished subjects showed a significantly lower systolic blood pressure, as well as a narrower pulse pressure, when compared with the control group. The diastolic blood pressure and resting heart rate were lower in the labourers; but this small difference was not statistically significant.

Cardiovascular responses to increasing doses of isoproterenol in the two groups is shown 


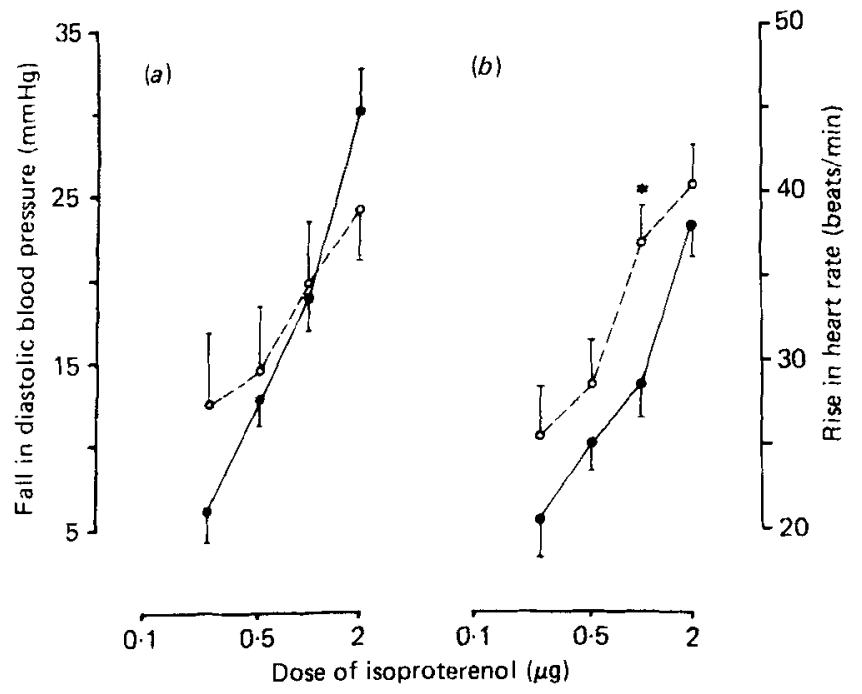

Fig. 1. Comparison of cardiovascular responses $((a)$ fall in diastolic blood pressure and $(b)$ chronotropic response) to increasing intravenous single doses of isoproterenol of undernourished Indian adult male subjects $(O)$ with those of normal-weight controls $(O) .{ }^{*} P<0.05$. Points are mean values with their standard errors represented by vertical bars.

in Fig. 1. The undernourished subjects showed significantly greater positive chronotropic responses to isoproterenol at the $1.0 \mu \mathrm{g}$ dose $(P<0.05)$ when compared with the normalweight group. The $\mathrm{CD}_{25}$ of the undernourished group $(0.37$ (SE 0.06$) \mu \mathrm{g}$ ) was $43 \%$ lower than that of the normal-weight controls $(0.65$ (SE 0.13$) \mu \mathrm{g})$ although this difference was not statistically significant. Diastolic blood pressure decrements were also greater but not statistically significant in the undernourished subjects. The mean diastolic blood pressure fall of 11.2 (SE $2 \cdot 3) \mathrm{mm} \mathrm{Hg}$ at the $\mathrm{CD}_{25}$ in the undernourished group was similar to the mean of the control subjects, i.e. $12 \cdot 2$ (SE $2 \cdot 4) \mathrm{mm} \mathrm{Hg}$.

Two-way analysis of variance (ANOVA) of the values for dose responses in control and undernourished subjects indicated that all subjects in both groups showed increasing responses with four graded doses of isoproterenol in both the chronotropic response (sum of squares $2989 \cdot 1,3 \mathrm{df}$, variance ratio $F 24.7 ; P<0.005$ ) and diastolic blood pressure fall (sum of squares $3451.7,3 \mathrm{df}$, variance ratio $F 13.7 ; P<0.001$ ). The ANOVA of dose responses between the two groups was also statistically significant for the chronotropic response (sum of squares $284.9,1 \mathrm{df}$, variance ratio $F 7.1 ; P<0.05$ ) but not for the decrement in diastolic pressure (sum of squares $33.0,1 \mathrm{df}$, variance ratio $F 0.4 ; P>0.05$ ).

The undernourished subjects who had significantly lower body-weights than the normalweight controls (Table 1) received identical doses of isoproterenol. To exclude the possibility that the greater increase in heart rate and larger drop in diastolic blood pressure seen in the undernourished group was due to the higher dose administered per $\mathrm{kg}$ body-weight as compared with the normal weight controls, correlation coefficients were calculated between the changes in heart rate or diastolic blood pressure and body-weight or dose per kg bodyweight of the subjects. No correlations of any significance were observed within each group either between body-weight or dose per $\mathrm{kg}$ and the cardiovascular indices measured.

The cardiovascular responses of the undernourished and control subjects to HUT did not show any differences. On tilting to $70^{\circ}$, the heart rate increased in all subjects; the increase in heart rate over the resting heart rate was, however, not different between the two 
groups. The maximum increase in heart rate (bpm) which occurred between 5 and 10 min of HUT was approximately 25 and was similar in both groups. The mean increase in heart rate (bpm) was 24.9 (SE 2.5) at $8 \mathrm{~min}$ in controls and 23.7 (SE 2.7) at $8 \mathrm{~min}$ in the undernourished. Within $1 \mathrm{~min}$ of tilting to $70^{\circ}$ the systolic blood pressure dropped and the diastolic blood pressure increased in all subjects, in both groups. Changes in systolic and diastolic blood pressures and pulse pressure appeared to be similar in the two groups, both during and after the tilt.

\section{DISCUSSION}

The undernourished subjects in the present study showed an increased cardiac $\beta$-adrenoceptor responsiveness when compared with normal-weight controls. They had lower energy intakes and lower anthropometric indices. Undernutrition or energy restriction is known to cause a marked reduction in sympathetic drive as indicated by reduced turnover of catecholamines in different tissues in experimental animals (Young \& Landsberg, $1977 \mathrm{~b}$, 1979). A reduction in circulating levels of catecholamines and a fall in the excretion of catecholamine metabolites occurs even in human subjects who are semi-starved (Jung et al. 1979; Shetty et al. 1979). A diminished sympathetic drive manifested by a reduced turnover as well as reduced circulating levels of catecholamines, is usually associated with the development of supersensitivity to agonist stimulation or up-regulation of adrenoceptor numbers (Motulsky \& Insel, 1982). Depletion of catecholamines or treatment with adrenergic antagonists leads to supersensitivity of tissues to catecholamines associated with an up-regulation of adrenoceptor numbers, best studied in man during the administration and withdrawal of the $\beta$-adrenergic antagonist propranolol (Gerber \& Nies, 1979; Aarons et al. 1980; Fraser et al. 1981). A diet-induced lowering of sympathetic activity might thus explain the enhanced adrenergic responsiveness observed in these undernourished subjects. This may also probably be akin to the type of adrenergic hypersensitivity seen in patients with sympathetic denervation (Davies et al. 1982). In these patients low levels of the endogenous neurotransmitter noradrenaline are associated with increased $\alpha$-adrenergic receptor numbers and an enhanced pressor response to infused noradrenaline. Similar agonist regulation of adrenergic responsiveness is likely in the undernourished subjects who also show clinical evidence of a lowered sympathetic drive, manifested as a lower systolic blood pressure compared with normal-weight subjects.

Thyroid hormones have been shown to enhance adrenergic receptor numbers and sensitivity (Ginsberg et al. 1981). Both $\mathrm{T}_{4}$ and $\mathrm{T}_{3}$ cause increased myocardial catecholamine sensitivity by increasing $\beta$-receptor numbers (Williams et al. 1977). Altered energy intake is usually associated with changed levels of total serum $T_{3}$ and also free $T_{3}$; the latter considered to be the most sensitive indicator of biological activity of thyroid hormones (Danforth, 1983). All subjects were euthyroid with comparable free $T_{3}$ and total $T_{4}$ levels in the control and undernourished groups. Total $T_{3}$ levels were, however, lower in the undernourished group although this was not statistically significant. The lower total serum $\mathrm{T}_{3}$ level is probably the result of the lower energy intake of the undernourished group, since energy restriction or semi-starvation is known to lower $T_{3}$ levels (Chopra et al. 1975; Shetty et al. 1979). This decrease in total $T_{3}$ is also reflected in a lower $T_{3}: T_{4}$ value and significantly lower values have been reported by Chopra \& Smith (1975) in subjects with severe protein-energy malnutrition.

It has been shown that there are complex interactions between blood pressure, heart rate and vagal tone after a single dose of the $\beta$-agonist isoproterenol (Arnold \& McDevitt, 1983). The increase in heart rate observed following isoproterenol injection is caused by both a direct stimulation of cardiac $\beta$-adrenoceptors as well as by an indirect effect resulting 
from $\beta_{2}$-adrenoceptor-mediated peripheral vasolidation, with a consequent fall in arterial blood pressure and hence reflex withdrawal of cardiac vagal tone (Dunlop \& Shanks, 1968; Arnold \& McDevitt, 1983). In the present study, the undernourished subjects showed a fall in diastolic blood pressure following isoproterenol, comparable to the response observed in the normal-weight group. The degree of vagal withdrawal could possibly have been greater in the undernourished group, which might have further enhanced the cardiac acceleration seen following isoproterenol. This might imply a greater vagal tone in such undernourished individuals. Another study done by us (Shetty \& Jayarajan, 1987) on the effects of vagal blockade, using intravenous atropine, in undernourished and normalweight subjects has shown that undernourished subjects have a greater vagal tone compared with normal-weight controls. Thus the greater cardiac response observed in undernourished individuals in response to isoproterenol might be both the direct effect of enhanced $\beta$ adrenoceptor responsiveness as well as the indirect effect of the reflex withdrawal of an excess vagal tone, due to the fall in blood pressure.

Sex, body-weight, body surface area and the resting heart rate differences do not seem to influence the responses of the single-dose isoproterenol sensitivity test (Cleaveland et al. 1972). Results from twenty-seven dose-response curves of fifteen subjects with a wide range of body-weights (46-87.2 kg) also confirmed that body-weight is not a factor that influences the response to isoproterenol (George et al. 1972). The absence of any association between body-weights or dose per $\mathrm{kg}$ body-weight and the changes in both the cardiovascular indices in the present study, indicated that the differences in responses between the undernourished and normal-weight groups were not the results of undernourished subjects having lower body-weights and receiving a marginally higher dose per $\mathrm{kg}$ body-weight of isoproterenol. Age seems to be the only factor that influences the dose response to isoproterenol; subjects of age range 20-35 years showing decreasing sensitivity with increasing age (Cleaveland et al. 1972). The undernourished adult males who were slightly older than their normal weight controls were expected to have a reduced responsiveness, if any, due to age. The increased responsiveness in the undernourished group was therefore not likely to be related to either body-weight or age differences.

In conclusion, the undernourished subjects on lowered energy intakes showed an enhanced myocardial $\beta$-adrenergic receptor sensitivity. Our findings imply a denervation type of supersensitivity, associated with a diet-related reduced catecholaminergic drive. The latter would result in diminished synthesis and turnover of the sympathetic neurotransmitter noradrenaline. The positive chronotropic response may be further potentiated by the reflex withdrawal of an overactive vagal effect as a result of the drop in blood pressure induced by the stimulation of vascular $\beta_{2}$-adrenoceptors by isoproterenol. Despite the increased cardiovascular receptor responsiveness, there appears to be no compromise of the physiological response to change in posture, suggesting that the lowered sympathetic outflow may be compensated by an increase in responsiveness in order to maintain optimal cardiovascular functioning during undernutrition. This may explain why subjects who have to do physical labour appear to be apparently healthy and show good cardiovascular performance despite restricted intakes of energy (Shetty, 1984).

The present study was supported by a grant-in-aid from the Wellcome Trust, UK and the Medical Research Centre, Bombay Hospital Trust, to P. S. S. 


\section{REFERENCES}

Aarons, R. D., Nies, A. S., Gal, J., Hegstrand, L. R. \& Molinoff, P. B. (1980). Journal of Clinical Investigation 65, 949-957.

Arnold, J. M. O. \& McDevitt, D. G. (1983). British Journal of Clinical Pharmacology 15, 423-429.

Bristow, M. R., Ginsberg, R., Minobe, W., Cubicciotti, R. S., Sageman, W. S., Lurie, K., Billingham, M. E., Harrison, D. C. \& Stinson, E. B. (1982). New England Journal of Medicine 307, 205-211.

Chopra, I. J., Chopra, U., Smith, S. R., Reza, M. \& Solomon, D. H. (1975). Journal of Clinical Endocrinology and Metabolism 41, 1043-1049.

Chopra, I. J. \& Smith, S. R. (1975). Journal of Clinical Endocrinology and Metabolism 40, 221-227.

Cleaveland, C. R., Rangno, R. E. \& Shand, R. G. (1972). Archives of Internal Medicine 130, 47-52.

Colucci, W. S., Alexander, R. W., Williams, G. H., Rude, R. E., Holman, B. L., Konstan, M. A., Wynne, J., Mudge, G. H. Jr \& Braunwald, E. (1981). New England Journal of Medicine 305, 185-190.

Crandall, D. L., Lai, M. F., Huggins, F. J., Tanikella, T. K. \& Cervoni, P. (1983). American Journal of Physiology 244, H444-H448.

Danforth, E. Jr (1983). American Journal of Clinical Nutrition 38, 1006-1017.

Davies, B., Sudera, D., Sagnella, G., Marchesi-Saviotti, M., Mathias, C., Bannister, R. \& Sever, P. (1982). Journal of Clinical Investigation 69, 779-784.

Dunlop, D. \& Shanks, R. G. (1968). British Journal of Pharmacology and Chemotherapy 32, 201-218.

Durnin, J. V. G. A. \& Womersley, J. (1974). British Journal of Nutrition 32, 77-92.

Fraser, J., Nadeau, J., Robertson, D. \& Wood, A. J. J. (1981). Journal of Clinical Investigation 67, $1777-1784$.

George, C. F., Conolly, M. E., Fenyvesi, T., Briant, R. \& Dollery, C. T. (1972). Archives of Internal Medicine 130, 361-364.

Gerber, J. G. \& Nies, A. S. (1979). New England Journal of Medicine 301, 1234-1235.

Ginsberg, A. M., Clutter, W. E., Shah, S. D. \& Cryer, P. E. (1981). Journal of Clinical Investigation 67, 17851791.

Jung, R. T., Shetty, P. S., Barrand, M. A., Callingham, B. A. \& James, W. P. T. (1979). British Medical Journal i, 12-13.

Lefkowitz, R. J., Caron, M. G. \& Stiles, G. L. (1984). New England Journal of Medicine 310, 1570-1579.

Motulsky, H. J. \& Insel, P. A. (1982). New England Journal of Medicine 307, 18-29.

Shetty, P. S. (1984). Human Nutrition: Clinical Nutrition 38C, 443-451.

Shetty, P. S. \& Jayarajan, M. P. (1987). Proceedings of the VI Annual Conference of the Indian Association of Biomedical Scientists (In the Press).

Shetty, P. S., Jung, R. T. \& James, W. P. T. (1979). Lancet i, 77-79.

Williams, L. T., Lefkowitz, R. J., Watanabe, A. M., Hathway, D. R. \& Besch, H. R. (1977). Journal of Biological Chemistry 252, 2787-2789.

Young, J. B. \& Landsberg, L. (1977 a). Nature 269, 615-617.

Young, J. B. \& Landsberg, L. (1977 b). Science 196, 1473-1475.

Young, J. B. \& Landsberg, L. (1979). American Journal of Physiology 236, E524-E533. 